\title{
Why Early Diagnosis Is Better for Alzheimer's Patients
}

\author{
Thierry Voisin,,$^{1,2}$ Sandrine Sourdet, ${ }^{1,2}$ Julien Delrieu ${ }^{1,2}$ and Bruno Vellas ${ }^{1,2}$ \\ 1. Alzheimer's Disease Clinical Research Centre, Gerontopôle, Toulouse University Hospital; 2. INSERM U 558, Toulouse
}

DOI:10.17925/ENR.2009.04.01.10

\begin{abstract}
Alzheimer's disease (AD) is the most common cause of dementia. AD progression leads to a significant deterioration in cognitive function, resulting in a significant impact not only on patient quality of life but also on the quality of life of family members/care-givers. Some physicians do not feel that diagnosing $A D$ is necessary due to the absence of any cure and the stress related to diagnosis. Nevertheless, it is important to diagnose $A D$ as early as possible. Early diagnosis of $A D$ allows the patient to be more involved in treatment planning, since at this stage the patient's cognitive function will be near normal. Many forms of the disease do not progress rapidly, and early diagnosis and treatment will allow a good quality of life for the patient, family members and care-givers. The general practitioner has a special role in detecting and following up patients diagnosed with $\mathrm{AD}$.
\end{abstract}

\section{Keywords}

Alzheimer's disease ( $\mathrm{AD}$ ), diagnosis, early, issues, dementia, mild cognitive impairment (MCl)

Disclosure: Thierry Voisin, Sandrine Sourdet and Julien Delrieu have no conflicts of interest to declare. Bruno Vellas has grants pending from Pfizer and Eisai. No sources of funding were used to assist in the preparation of this article.

Received: 25 June 2009 Accepted: 4 August 2009

Correspondence: Bruno Vellas, Alzheimer's Disease Clinical Research Centre, Gerontopôle, CHU Toulouse, France, Toulouse University Hospital, 170 Ave de Casselardit, 31300 Toulouse, France. E: vellas.v@chu-toulouse.fr

Support: The publication of this article was funded by an educational grant from Eisai Europe Limited. The views and opinions expressed are those of the authors and not necessarily those of Eisai Europe Limited.

Alzheimer's disease (AD) is the most common cause of dementia, with an estimated prevalence of more than 106 million worldwide by $2050 .{ }^{1}$ It is a neurodegenerative disease characterised by the accumulation of senile plaques (deposits of the $\beta$-amyloid peptides) and neurofibrillary tangles (abnormal hyperphosphorylated insoluble forms of the tau protein). Patients with AD often experience problems of memory loss, confusion, impaired judgement, disorganised thinking, loss of ability to express themselves and disorientation with regard to time, space and location. AD progression leads to a significant deterioration in cognitive function, resulting in patients being increasingly bedridden and dependent on care-givers and, eventually, professional long-term care (LTC) such as nursing home care. AD has a substantial impact not only on the quality of life of ageing people, but also on the lives of family members and care-givers. The burden of $A D$ is expected to substantially increase as the baby-boomer generation ages, with age $\geq 65$ years being a major risk factor for the development of AD. This places an increased emphasis on the need to evaluate if and when patients with signs and/or symptoms of $A D$ should be diagnosed. According to the French Personnes Agees Quid (PAQUID) cohort study, the Mini-Mental State Examination (MMSE) score at the time of diagnosis of dementia for the majority of cases is $16-20,{ }^{2}$ so the diagnosis is established at the moderate stage of the disease in the majority of cases. In Europe, the average time required to establish the diagnosis of dementia (time between the onset of symptoms of the disease and diagnosis) is estimated at 20 months, which underlines the current level of late diagnosis. ${ }^{3}$ In 112 cases of dementia detected by general practitioners (GPS), the documentation rate was $33 \%$ for the mild stage of dementia, whereas it was 46 and $73 \%$, respectively, for the moderate and severe stages. ${ }^{4}$ Furthermore, in many cases the initial symptoms do not alert the patient or relatives, as there is frequent confusion between ageing and dementia; also, when patients see a doctor, dementia is often not identified by the GP, which may be due to problems such as difficulties in distinguishing between a degenerative condition and cognitive recall with sensory problems or general conditions, or because of poor-quality information for practitioners relating to specifics about $A D$ and the treatment to prescribe, etc. It is therefore important to provide GPS with information and practical tools that will help them recognise the symptoms that may indicate dementia and to look for the signs requiring referral to a specialist for assessment. In fact, improving the diagnostic process in primary care represents a key step in increasing the number of cases detected in the population.

For some physicians and other health professionals, the clinical diagnosis of $A D$ presents a dilemma. Some physicians may feel that the stress related to a confirmed diagnosis may have a negative effect on the patient, especially as there are currently no curative treatments available. However, a lack of diagnosis can create a dangerous situation, as without any diagnosis most of these patients are likely to face several problems related to the symptoms of $A D$, such as poor 
nutritional intake, weight loss, increased side effects due to mistakes in medication use and reduced quality of life. The physician could even be held responsible for any possible problems. Furthermore, the development of $A D$ has been shown to follow a predictable pattern, with the changes taking place years before the onset of clinical symptoms. ${ }^{5,6}$ These findings, plus the knowledge that many forms of the disease progress slowly, suggest that early diagnosis and subsequent intervention would allow an opportunity to achieve a better quality of life for the patient, family members and care-givers for a longer period of time. In view of these issues, it seems valid to make the diagnosis of $A D$ as early as possible as it would enable better management of the disease through pharmacological or nonpharmacological care. The progress of patients and any complications could also then be monitored by long-term follow-up, with appropriate early assistance such as defined in the Specific Care and Assistance Plan for Alzheimer's Disease (PLASA) study.? The oldest AD sufferers are expected to be prone to the greatest problems and should be given all the help needed as soon as possible.

\section{Diagnostic Criteria for Alzheimer's Disease}

Diagnosis of $A D$ is based on the prevailing criteria set out by either the National Institute of Neurological Disorders and Stroke-Alzheimer Disease and Related Disorders (NINDS-ADRDA) working group, ${ }^{8}$ or the American Psychiatric Association's Diagnostic and Statistical Manual of Mental Disorders, 4th edition (DSM-IV-TR). ${ }^{9}$ The diagnostic process consists of two steps: initial identification of a dementia component followed by the application of criteria based on the clinical features of the AD phenotype. In recent years, understanding of the biological basis of $A D$ has greatly improved and distinctive biomarkers of the disease have been identified. These biomarkers include structural brain changes visible on magnetic resonance imaging (MRI), ${ }^{10}$ molecular neuroimaging changes seen with positron emission tomography (PET) ${ }^{11}$ and changes in cerebrospinal fluid biomarkers. ${ }^{12}$ This progress in the elucidation of the development of $A D$ and identification of biomarkers of disease has led to the proposal of revised diagnostic criteria for AD. ${ }^{13}$ These criteria aim to include both the earliest stages of disease, i.e. before confirmed dementia, and the complete range of disease stages. The core diagnostic criteria focus on the presence of early and significant episodic memory impairment. This should be complemented by the presence of at least one abnormal biomarker among structural neuroimaging with MRI, molecular neuroimaging with PET and cerebrospinal fluid analysis of amyloid or tau proteins.

\section{Issues Associated with a Lack of Diagnosis}

some physicians and other health professionals do not feel that diagnosing $A D$ is necessary due to the absence of any cure and the stress related to diagnosis. According to this school of thought, a diagnosis would not be too useful as it would lead to fear and emotional distress for patients, serving only to compound the patient's misery of knowing they have the disease yet knowing there is no curative agent available. However, the counter-argument is that this assumption is not valid, as many patients exhibit a great desire for an early diagnosis, with up to $80 \%$ of older adults wishing to know as early as possible whether they have probable or definitive AD. ${ }^{14,15} \mathrm{~A}$ recent examination of short-term changes in depression and anxiety after receiving a dementia diagnosis, using a 15-item Geriatric Depression Scale and a 20-item 'state' version of the State-Trait Anxiety Inventory, noted that no significant changes in depression occurred in individuals or their companions, regardless of diagnostic outcome or dementia severity. ${ }^{16}$
The lack of a diagnosis of probable or definitive $A D$ can have potentially deleterious consequences for the patient, as disease progression can lead to several problems, particularly in those with advanced stages of the disease with a low cognitive function score, e.g. an MMSE score $<15$. Such a low score has implications for the patient's safety. Both the Réseau sur la maladie d'Alzheimer Français (REAL.FR) study ${ }^{17}$ and the Impact of Cholinergic Treatment Use (ICTUS) study ${ }^{18}$ observed that more than $19 \%$ of patients with $A D$ live alone. This observation suggests a greater chance of patients with cognitive impairment experiencing medication compliance issues and/or making mistakes with medications. This in turn can lead to increased risks of side effects or aggravation of co-existing diseases when treatments are not taken. Memory loss is one of the most common symptoms of $A D$ and has a significant impact on the daily routine of a patient. Memory loss will also affect a patient's ability to manage personal and financial activities. $A D$ could also have an impact on the nutritional intake of the affected person due to difficulties in carrying out shopping and cooking activities, which have been linked to a poor Instrumental Activities of Daily Living scale (IADL) score. AD could also have wider implications; for example, in cases where the patient is also a care-giver with responsibilities for a partner or family member who also has health issues, $A D$ may have a negative impact on the provision of care. Advancing AD could also have a significant negative impact on the care-giver, as deteriorating disease leads to a decrease in cognitive function of the patient and increased dependence on the care-giver.

\section{Understanding the Importance of an Earlier Diagnosis}

Increasing evidence shows that $A D$ progresses slowly during the early phase of the disease and that the disease evolves along a predictable pattern of progression in the brain, ${ }^{5,6}$ with the molecular pathomechanisms of $A D$ becoming active many years before neurons start dying and clinical symptoms appear. ${ }^{19}$ since there would be a lower burden of amyloid and hyperphosphorylated tau, little or no deterioration in cognitive function in the early stages of the disease and a slow rate of disease progression, an earlier diagnosis followed by symptomatic or disease-modifying therapy could potentially be an effective strategy to help maintain a good quality of life for patients, family members and care-givers. The most commonly used agents for early AD are the cholinesterase inhibitors, including donepezil, rivastigmine and galantamine, which have been shown to improve cognitive and global function in some patients.

The early diagnostic approach has also provided primary care physicians with an opportunity to offer early psychosocial support directly to individuals affected by AD. Psychosocial support can be offered to early-stage groups, and the opportunity to share experiences and increased social support have been reported among the benefits of such an approach. ${ }^{20}$ While there are few trained professionals and a lack of psychosocial support groups, data indicate that psychotherapy of early-stage individuals could help manage the disease and reduce depression, ${ }^{21}$ and such an approach should be considered in the future.

Patients with $A D$ have low functional disability and relatively good cognitive function in the early stages. Therefore, these patients can be involved in treatment planning, expressing opinions and desires regarding how to improve and maintain quality of life. ${ }^{22}$ Indeed, 
patients with early-stage $A D$ are increasingly requesting involvement in future treatment planning. ${ }^{23}$ Among the cognitive domains, the level of awareness affects the efficacy of some interventions. ${ }^{24}$ As this cognitive function deteriorates with advancing disease, a diagnostic and interventional strategy could be beneficial if carried out quite early in the disease process, when the level of awareness is near normal. By contrast, if the diagnosis is not made at all or only when the patient experiences severe health problems, the patient would have various complications, for example aggression, agitation, delusions, hallucinations and weight loss. At this point, the family would be confused and would not be able to understand the patient's condition or why the diagnosis was not made earlier. This could also cause problems for the physician; for instance, the family could blame or question the physician's decision to make a diagnosis only when the patient's health had significantly deteriorated.

Patients with $A D$, their family members and their care-givers can be placed under great emotional and financial burden due to the patient's declining cognitive and functional abilities. The burden on family members and care-givers, plus the deteriorating cognitive ability of the patient, often leads to the latter requiring formal LTC services, which can be costly to the patient and family members. Instead, if $A D$ is diagnosed as early as possible, there would be a high chance of managing disease progression and reducing the symptoms, leading to either delayed or reduced need for entry into nursing homes and consequently cost savings for the patient and family members. Other benefits would include improved economic efficiency and better quality of life for the patient and the individuals supporting him or her. In one study, Monte Carlo cost-benefit analyses evaluated the costs and benefits of the early identification and treatment of AD patients. ${ }^{25}$ The analyses used LTC cost data from Wisconsin and data about the potential benefits of pharmacological and non-pharmacological therapies (e.g. care-giver support). The net benefits have been shown to be the highest when cases are identified at earlier stages, e.g. at an MMSE score of $28,{ }^{25}$ and when drug therapy is combined with a care-giver intervention programme. ${ }^{26}$ Additionally, mean net social benefits of US $\$ 94,000$ were reported for 10,000 trials for a particular Monte Carlo analysis, assuming a drug treatment effect (MMSE/L) for a 70-year-old married woman with a starting MMSE score of $26 .{ }^{26}$ These analyses show that early detection of $A D$ followed by pharmacological intervention and care-giver support results in large, positive net social benefits. These findings also indicate that the attitude of healthcare professionals towards $A D$ will have to change, with physicians aiming to diagnose and treat $A D$ as early as possible, paying particular attention to the oldest sufferers as they are most likely to experience the greatest problems and be the largest consumers of LTC services.

\section{Conclusions}

While some physicians and health professionals may be reluctant to diagnose $A D$, this lack of diagnosis can result in numerous problems related to the symptoms of disease, with advancing disease resulting in significant deterioration in clinical symptoms, dependence on caregivers and the need for professional LTC services. Diagnosing $A D$ as early as possible will allow the opportunity to achieve a good quality of life for patients, family members and care-givers for a longer period of time. For all of these reasons, it is important to diagnose $A D$ early in the course of the disease, regardless of the patient's age. Once detected, AD can be managed by the medical staff, the patient and the family circle. The worst situation would be to handle impairment, suffering and end-of-life care for an unknown diagnosis. The GP has a special role in detecting and following up patients with $A D$, especially the oldest of the aged patients. A task force discussed the importance of an early diagnosis of $A D$ at the world congress of the International Association of Gerontology and Geriatrics in July 2009. The speakers provided an update on biomarkers, imaging technologies and the usefulness of various earlystage diagnostic tools as part of therapeutic monitoring, and discussed neuroradiological biomarkers that could enable assessment of disease progression during clinical trials using new compounds that target amyloid protein or other lesions.
1. Brookmeyer $R$, Johnson $E$, Ziegler-Graham $K$, et al., Forecasting the global burden of Alzheimer's disease, Working Paper, 2007;130.

2. Ramaroson $\mathrm{H}$, Helmer $\mathrm{C}$, Barberger-Gateau P, et al., Prevalence of dementia and Alzheimer's disease among subjects aged 75 years or over: updated results of the PAQUID cohort, Rev Neurol (Paris), 2003;159:405-11.

3. Bond J, Stave C, Sganga A, et al., Inequalities in dementia care across Europe: key findings of the Facing Dementia Survey, Int J Clin Pract Suppl, 2005;8-14.

4. Lopponen $\mathrm{M}$, Raiha I, Isoaho $\mathrm{R}$, et al., Diagnosing cognitive impairment and dementia in primary health care-a more active approach is needed, Age Ageing, 2003;32:606-12.

5. Braak $H$, Braak E, Neuropathological stageing of Alzheimer-related changes, Acta Neuropathol, 1991;82: 239-59.

6. Delacourte A, David JP, Sergeant N, et al., The biochemical pathway of neurofibrillary degeneration in aging and Alzheimer's disease, Neurology, 1999;52:1158-65.

7. Nourhashemi F, Gillette-Guyonnet S, Andrieu S, et al., A randomized trial of the impact of a specific care plan in 1120 Alzheimer's patients (PLASA Study) over a two-year period: design and baseline data, J Nutr Health Aging, 2008;12:263-71.

8. McKhann G, Drachman D, Folstein M, et al., Clinical diagnosis of Alzheimer's disease: report of the NINCDSADRDA Work Group under the auspices of Department of Health and Human Services Task Force on Alzheimer's Disease, Neurology, 1984;34:939-44.

9. American Psychiatric Association, Diagnostic and statistical manual of mental disorders (IV-TR), 4th edn-text revised, 2000.

10. Jack $C R$, Jr., Dickson DW, Parisi JE, et al., Antemortem MRI findings correlate with hippocampal neuropathology in typical aging and dementia, Neurology, 2002;58:750-57.

11. Silverman $\mathrm{DH}$, Gambhir SS, Huang HW, et al., Evaluating early dementia with and without assessment of regiona cerebral metabolism by PET: a comparison of predicted costs and benefits, J Nucl Med, 2002:43:253-66.

12. Hulstaert F, Blennow $K$, Ivanoiu $A$, et al., Improved discrimination of AD patients using beta-amyloid(1-42) and tau levels in CSF, Neurology, 1999;52:1555-62.

13. Dubois B, Feldman HH, Jacova C, et al., Research criteria for the diagnosis of Alzheimer's disease: revising the NINCDS-ADRDA criteria, Lancet Neurol, 2007:6:734-46.

14. Dale W, Hemmerich J, Hill EK, et al., What correlates with the intention to be tested for mild cognitive impairment (MCl) in healthy older adults? Alzheimer Dis Assoc Disord, 2008;22:144-52.

15. Dale W, Hougham GW, Hill EK, et al., High interest in screening and treatment for mild cognitive impairment in older adults: A pilot study, J Am Geriatr SOC, 2006;54: 1388-94.

16. Carpenter $\mathrm{BD}$, Xiong $\mathrm{C}$, Porensky EK, et al., Reaction to a dementia diagnosis in individuals with Alzheimer's diseas and mild cognitive impairment, I Am Geriatr SOC, 2008;56: 405-12.

17. Balardy L, Voisin T, Cantet $C$, et al., Predictive factors of emergency hospitalisation in Alzheimer's patients: results of one-year follow-up in the REAL.FR Cohort, J Nutr Health Aging, 2005:9:112-16.
18. Reynish E, Cortes F, Andrieu S, et al., The ICTUS Study: A Prospective longitudinal observational study of 1,380 AD patients in Europe. Study design and baseline characteristics of the cohort, Neuroepidemiology, 2007:29:29-38.

19. DeKosky ST, Marek K, Looking backward to move forward: early detection of neurodegenerative disorders, science, 2003;302:830-34.

20. Zarit SH, Femia EE, Watson J, et al., Memory Club: a group intervention for people with early-stage dementia and their care partners, Gerontologist, 2004;44:262-9.

21. Cheston R, Jones K, Gilliard J, Group psychotherapy and people with dementia, Aging Ment Health, 2003;7:452-61.

22. Vernooij-Dassen $M$, Derksen $E$, Scheltens $P$, et al., Receiving diagnosis of dementia: The experience over time, Dementia, 2006:5:397-410.

23. Young R, Medical experiences and concerns of people with Alzheimer's Disease. In: The Person with Alzheimer's Disease: Pathways to understanding the experience, 2002;29-46.

24. Clare L, Wilson BA, Carter G, et al., Awareness in earlystage Alzheimer's disease: relationship to outcome of cognitive rehabilitation, J Clin Exp Neuropsychol, 2004;26: 215-26.

25. Weimer DL, Sager MA, Early identification and treatment of Alzheimer's disease: social and fiscal outcomes, Alzheimers Dement, 2009;5:215-26.

26. Mittelman MS, Haley WE, Clay OJ, et al., Improving caregiver well-being delays nursing home placement of patients with Alzheimer disease, Neurology, 2006;67:1592-9. 